\title{
PAPER
}

\section{Hemisensory syndrome is associated with a low diagnostic yield and a nearly uniform benign prognosis}

\section{Toth}

Correspondence to:

Dr C Toth, Department of

Neurological Sciences,

Room 128, Heritage

Medical Research Building,

University of Calgary,

3330 Hospital Drive NW,

Calgary, Alberta, Canada

$\mathrm{T} 2 \mathrm{~N} 4 \mathrm{~N} 1$;

corytoth@shaw.ca

Received

3 December 2002

In revised form

5 February 2003

Accepted 4 March 2003

\begin{abstract}
Objective: To describe the diagnostic yield and prognosis for patients with hemisensory syndrome. Background: The aetiology, utility of diagnostic procedures, and outcome of hemisensory syndrome in patients with exclusive hemibody complaints having only subjective sensory abnormalities on examination is unknown.

Methods: Patients were prospectively identified with hemisensory syndrome in a tertiary care institution from 1998-2002. Diagnostic procedures were analysed for sensitivity and clinical follow up was performed.

Results: Thirty four patients, 25 (74\%) women, of age 35 (SD 1 1) years were identified. The hemisensory syndrome occurred on the left side in $23(68 \%)$ cases. Neuroimaging of the brain demonstrated diagnostic abnormalities representing ischaemic aetiology in one case. Other diagnostic testing including cerebrospinal fluid examination, electrophysiological testing, carotid ultrasonography, echocardiography, and blood testing revealed no diagnostic abnormalities. Sixteen patients $(47 \%)$ continued to complain of hemisensory difficulties after all investigations were completed at 9.6 (5.8) days. One patient with a history of systemic lupus erythematosus and positive antiphospholipid antibodies had a second event diagnosed as stroke seven months after presentation. Clinical follow up at 16 (7) months revealed persisting symptoms in $6(20 \%)$ of 30 patients. Six $(50 \%)$ of 12 patients agreeing to psychiatric assessment received diagnoses of personality or mood disorders.

Conclusions: Diagnostic yield in hemisensory syndrome is low, and prognosis is almost always uniformly benign. The author advocates careful assessment of medical history and consideration for neuroimaging in this group of patients.
\end{abstract}

$\mathrm{T}$ he aetiology of non-anatomical or non-dermatomal sensory deficits has been speculated upon for decades. Often, these have been attributed to "non-organic" causes. Jean-Martin Charcot first discussed hysterical hemianaesthesia as part of the presentation of the famous patient Justine Etchevery in 1872. ${ }^{1}$ Despite Charcot's diagnoses of hysteria in such cases, he spent much of his later career preoccupied with a search for a pathological explanation for hysterical phenomena, seemingly rejecting the defiance of such symptoms to neuroanatomical pathways. ${ }^{2}$ In 1885, Charcot asked "There is without doubt a lesion of the nervous centers, but where is it situated, and what is its nature?" ${ }^{3}$ in reference to patients with hysterical hemibody symptoms. Over the past century since the time of Charcot, diagnostic procedures for central nervous system disorders have improved considerably, permitting greater diagnostic testing in patients with possible neurological symptoms. What remains unknown is whether or not additional diagnostic testing in such cases provides a comparable improvement in diagnostic yield.

Non-organic sensory deficits have been described as being "diagnosed by their variability, sharp midline transition, and non-anatomic dermatomal distribution". ${ }^{4}$ Whether or not these characteristics can be applied to patients with hemibody sensory symptoms such as initially described by Charcot, which may have an anatomical basis in the absence of other medical conditions also has not yet been determined. Also, the cause and prognosis of patients with only hemibody sensory symptoms remains essentially unstudied.

I investigated the diagnostic yield and prognosis of patients presenting to a tertiary care centre with only hemisensory symptoms.

\section{METHODS}

Prospective identification of patients presenting to outpatient or emergency departments of a tertiary care centre in Saskatoon, Saskatchewan, Canada for the period of July 1998 to January 2002 was performed. The estimated size of the referral area for this tertiary care centre is 500 000. All patients complained of spontaneous onset of symptoms affecting one side of the body, including head and both limbs. Complaints could include any of numbness, sensory misperception, tingling, heaviness, weakness, or clumsiness. Patients with a history of presenting symptoms suggesting trauma, headache, or seizure were not considered. Patients with a past history of complex regional pain syndrome (CRPS), chronic pain syndrome, or issues with litigation were excluded. Physical examination was performed in all cases, and patients were excluded from the study if the examining neurologist found cognitive changes, motor findings, reflex abnormalities, or dyscoordination. Evidence of cortical sensory loss including extinction on double simultaneous sensory stimulation, agraphesthesia, or astereognosis also led to exclusion from the study. Other modalities of sensory examination performed included comparison of pinprick, light touch using a cotton stick, temperature sensation using a portion of cold metal, and vibratory threshold examination with a $128 \mathrm{~Hz}$ tuning fork over both sides of the body, as well as comparison of proprioception between both upper and lower extremities.

All patients were booked for investigations to be performed within 14 days of the onset of symptoms. Investigations

Abbreviations: $\mathrm{CT}$, computed tomography; MRI, magnetic resonance imaging; SLE, systemic lupus erythematosus; CRPS, complex regional pain syndrome 
performed included computed tomography (CT) and magnetic resonance imaging (MRI) of the brain, lumbar puncture with cerebrospinal fluid (CSF) examined for cell count, protein, cytology, and electrophoresis, carotid ultrasonography, echocardiography, electroencephalography (EEG), somatosensory and visual evoked potentials, and transcranial Doppler ultrasonography with bubble testing to examine for presence of a cardiopulmonary shunt. MRI sequences used included $\mathrm{Tl}$ and $\mathrm{T} 2$ weighted imaging, diffusion weighted imaging, and proton density imaging. Blood tests including complete blood count, electrolytes, urea, creatinine, serum glucose, creatine kinase, calcium, magnesium, thyroid stimulating hormone level, and vitamin B12 levels were performed in all patients. All patients were suggested to have psychiatric assessment during the study.

After initial investigations, diagnosis, and treatment if necessary, patients received follow up with a repeated history and physical examination after investigations were complete. Long term follow up was conducted between 6 to 18 months after completion of investigations either by a history taken by telephone or by outpatient assessment. Physical examination was performed for those patients seen in outpatient assessments as well as for those who complained of symptoms during the telephone interview.

\section{RESULTS}

A total of 34 patients, 25 (74\%) women, were identified over the 42 month period. The mean (SD) age of the patients was 35 (11) years. The symptomatic side of the body was the left side in 23 cases $(68 \%)$. The duration of symptoms prior to assessment was an average of 1.6 (1.2) days.

Initial symptoms consisted of unilateral numbness only in 12 patients $(34 \%)$, unilateral numbness and tingling in 10 patients $(28 \%)$, unilateral mixed sensory changes with perception of unilateral body heaviness in nine patients $(25 \%)$, unilateral sensory changes with perception of unilateral body heaviness and clumsiness in three patients $(8 \%)$, and unilateral tingling only in two patients (5\%). Onset was described as maximal at the start of symptoms in $12(35 \%)$ of patients and as gradually progressive over hours to days in remaining patients $(65 \%)$. Intermittent blurring of vision in the ipsilateral eye (asthenopia) was described by 10 patients $(28 \%)$ and ipsilateral disturbance of hearing was described by six patients $(16 \%)$.

Medical history was remarkable for migraine in six patients (18\%), hypertension in six patients $(18 \%)$, treated hypothyroidism in two patients $(6 \%)$, hyperlipidaemia in one patient $(3 \%)$, systemic lupus erythematosus (SLE) in one patient $(3 \%)$, a prior incidence of undiagnosed hemisensory syndrome in one patient $(3 \%)$, generalised epilepsy in one patient $(3 \%)$, and prior pseudoseizures in one patient $(3 \%)$. Psychiatric history was remarkable for major depression in nine patients $(26 \%)$, prior personality disorder in six patients (18\%) (borderline diagnosis in thee patients $(9 \%)$, histrionic personality disorder in two patients $(6 \%)$, and avoidant personality disorder in one patient $(3 \%))$, generalised anxiety disorder in two patients $(6 \%)$, dysthymic disorder in one patient $(3 \%)$, conversion disorder in one patient $(3 \%)$, and bipolar affective disorder in one patient (3\%).

All patients examined were found to have reduced perception of pinprick and light touch over the symptomatic side. Twenty three patients (68\%) identified an increased vibratory threshold relative to the asymptomatic side when vibratory stimulus was placed at the digits, sternum, and frontal bone. Three patients (9\%) showed loss of proprioception at digits of the upper and lower extremities on the symptomatic side as compared with the asymptomatic side. No patients identified a loss of temperature perception over the symptomatic side. Comparison of pinprick over the torso showed an abrupt border between abnormal pinprick sensation on the symptomatic side and normal pinprick sensation over the asymptomatic portions in $27(79 \%)$ of patients.

All 34 patients had blood tests, CT imaging of the brain, MRI of the brain, and carotid ultrasonography. Additional diagnostic procedures were performed in less than $100 \%$ of patients and are shown in table 1. Both the neurologist and patient knew any abnormalities of investigations before follow up assessment in all cases. Abnormalities found within blood tests in five patients (15\%) were deemed non-contributory. Further testing was performed in one patient $(3 \%)$ with a diagnostic CT scan finding and revealed a positive antiphospholipid antibody profile.

CT of the brain was abnormal in four patients ( $12 \%)$ and in one case, the abnormal finding was deemed significant-a single abnormal hypodensity in the corona radiata contralateral to the hemisensory loss. This patient had a history of SLE and presented with unilateral hemibody numbness only. MRI revealed abnormalities in the same four patients found to have CT scan abnormalities, with again one patient having an abnormality deemed to be significant-a single oval $\mathrm{T} 2$ and proton density hyperintensity in the corona radiata contralateral to the hemibody sensory disturbance, in the same location as was noted on this patient's CT scan. Diffusion weighted MRI similarly demonstrated a hyperintense lesion in this patient.

After completion of investigations, all patients had a follow up history and physical examination performed an average of 9.6 (5.8) days after presentation. Of the 34 patients, 16 patients $(47 \%)$ continued to complain of hemisensory difficulties. Physical examination at that time showed reduced perception of pinprick and light touch in all 16 patients, with raised vibratory threshold on the symptomatic side relative to asymptomatic side in 11 patients (32\% of all patients), all of whom had reported this deficit at presentation. The remainder of examination was normal in all patients at this initial follow up period.

Long term follow up by telephone was available for 30 $(88 \%)$ of 34 patients at 16 (7) months. Persisting symptoms were identified in 6 of 30 patients $(20 \%)$, including unilateral numbness in three patients $(10 \%)$ and unilateral tingling in three patients $(10 \%)$. One of the six patients had a second clinical syndrome consisting of right hemiparesis diagnosed as stroke that occurred seven months after first presentation. This patient was the same person identified with positive neuroimaging previously and a history of SLE. Since initial assessment, the patient had been found to have positive IgG lupus anticoagulant antibodies with a titre of 1:400, and was subsequently treated with anticoagulation after the occurrence of stroke with no further events over the following 13 months. No other patients developed any discrete neurological events during long term follow up. Examination was performed in a total of 10 patients, including all six patients that had complained of persisting symptoms. Examinations in asymptomatic patients were normal in all four cases. Two of the six $(33 \%)$ symptomatic patients seen at $12(5)$ months after initial assessment had hemisensory pinprick and light touch loss relative to their asymptomatic side, with the remainder of their examination normal. The two patients with abnormal examinations at this time continued to have a very sharp transition between abnormal and normal regions of pinprick sensation.

Physicians other than the author made subsequent diagnoses of migraine without aura (4), migraine with aura (2), major depression (2), generalised anxiety disorder (1), and ipsilateral carpal tunnel syndrome (1) in these patients over the course of follow up. Twelve (35\%) of 34 patients agreed to psychiatric assessments after investigation completion. Of those 12 patients agreeing to psychiatric assessment, six $(50 \%)$ had no psychiatric diagnosis. In the remaining six patients, two were diagnosed with histrionic personality disorder, two with borderline personality disorder, one with major depression, and one with dysthymic disorder. 
Table 1 Investigations performed and their result in patients with hemisensory syndromes

\begin{tabular}{|c|c|c|c|c|}
\hline Investigation & $\begin{array}{l}\text { Number of } \\
\text { patients with } \\
\text { investigation }\end{array}$ & $\begin{array}{l}\text { Number of } \\
\text { abnormal } \\
\text { investigations }\end{array}$ & Abnormalities of investigations & $\begin{array}{l}\text { Diagnostic } \\
\text { investigation } \\
\text { findings }\end{array}$ \\
\hline Blood tests & $34 / 34(100)$ & $5 / 34(15)$ & $\begin{array}{l}\text { Mild iron defiency anaemia } 2 / 34(6) \\
\text { Mild hypokalaemia } 1 / 34(3) \\
\text { Mild hypocalcaemia } 1 / 34(3) \\
\text { Transient creatine kinase increase } 1 / 34(3)\end{array}$ & None \\
\hline $\mathrm{CT}$ of brain & $34 / 34(100)$ & $4 / 34(12)$ & $\begin{array}{l}\text { Contralateral corona radiata hypodensity } 1 / 34 \text { (3) } \\
\text { Ipsilateral temporal fossa arachnoid cyst } 1 / 34 \text { (3) } \\
\text { Bilateral basal gangliar hypodensities } 1 / 34 \text { (3) } \\
\text { Ipsilateral frontal lobe cavenous angioma } 1 / 34 \text { (3) }\end{array}$ & $\begin{array}{l}\text { Contralateral corona } \\
\text { radiata hypodensity } \\
\text { in } 1 / 34 \text { consistent } \\
\text { with ischaemia }\end{array}$ \\
\hline MRI of brain & $34 / 34(100)$ & $4 / 34(12)$ & $\begin{array}{l}\text { Contralateral corona radiata hyperintensity } 1 / 34 \text { (3) } \\
\text { Ipsilateral temporal fossa arachnoid cyst } 1 / 34 \text { (3) } \\
\text { Bilateral basal gangliar hyperintensities } 1 / 34 \text { (3) } \\
\text { Ipsilateral frontal lobe cavenous angioma } 1 / 34 \text { (3) }\end{array}$ & $\begin{array}{l}\text { Contralateral corona } \\
\text { radiata } \\
\text { hyperintensity in } \\
1 / 34 \text { consistent with } \\
\text { ischaemia }\end{array}$ \\
\hline Carotid ultrasonography & $34 / 34(100)$ & $0 / 34(0)$ & None & None \\
\hline Transthoracic echocardiography & $29 / 34(85)$ & $1 / 29(3)$ & Mobile intra-atrial septum in $1 / 29$ (3) & None \\
\hline $\begin{array}{l}\text { Transcranial Doppler } \\
\text { ultrasonography }\end{array}$ & $28 / 34(82)$ & $6 / 28(21)$ & $\begin{array}{l}\text { Positive bubble testing in } 6 / 28 \text { (21) suggesting a patent } \\
\text { foramen ovale }\end{array}$ & None \\
\hline $\begin{array}{l}\text { Evoked potentials (visual and } \\
\text { somatosensory) }\end{array}$ & $28 / 34(82)$ & $0 / 28(0)$ & None & None \\
\hline CSF examination & $28 / 34(82)$ & $0 / 28(0)$ & None & None \\
\hline EEG & $25 / 34(74)$ & $0 / 34(0)$ & None & None \\
\hline Transoesophageal echocardiography & $2 / 34(6)$ & $2 / 2(100)$ & Identification of patent foramen ovale in $2 / 2$ (100) & None \\
\hline Nerve conduction studies & $2 / 34(6)$ & $0 / 2(0)$ & None & None \\
\hline SPECT of brain & $1 / 34(6)$ & $0 / 1(0)$ & None & None \\
\hline
\end{tabular}

\section{DISCUSSION}

I report my experience with 34 patients who presented with hemisensory syndrome during a period of 42 consecutive months within a referral area having a population of 500000 . Thus, the estimated incidence of hemisensory syndrome in our geographical area is two patients per 100000 per year. This estimate is probably low, as not all patients with hemisensory syndrome would have necessarily been referred to this tertiary care institution during this time period.

An abrupt change in sensation, or exact splitting at the midline, was described by most (79\%) patients. Although organic disease has been reported as a cause of this pattern, ${ }^{56}$ cutaneous branches of the intercostal nerves typically overlap $1 \mathrm{~cm}-2 \mathrm{~cm}$, and sensory loss from an organic lesion would be expected to be paramedian. ${ }^{7}$ Most patients (68\%) in this study also demonstrated a difference in vibratory threshold between affected hemibody and unaffected side. Although commonly used by neurologists, examination using vibration over the left and right portions of a single midline bony structure such as the sternum or frontal bone is often described as asymmetric by patients with organic disease. ${ }^{68}$

Hemisensory deficits as presented in this study, can occur after pathological insults to the contralateral thalamus or parietal cortex. The thalamus, in particular, receives sensory input from the entire contralateral body, and lesions of the ventral posterior nuclear groups of the thalamus are associated with a contralateral hemisensory deficit of tactile, temperature, and pain sensation. ${ }^{9}$ Pure hemisensory deficit has been described with stroke of both ischaemic ${ }^{10}{ }^{11}$ and haemorrhagic ${ }^{12}$ aetiology. Neuroanatomical localisations that could produce a pure hemisensory deficit include the ventroposterior thalamus, ${ }^{13}{ }^{14}$ corona radiata, ${ }^{9}$ paramedian dorsalateral pons, ${ }^{10}$ lenticulocapsular region, ${ }^{912}$ and parietal lobe. ${ }^{11}$ Yet, aetiologies such as these were only found in one patient in this study. Further specialised diagnostic procedures may contribute to diagnosis in future patients, such as functional MRI (A Mailis, et al, American Pain Society, 2000), which has not demonstrated the expected activation of the contralateral somatosensory cortex with somatosensory stimulation of symptomatic limbs in patients with chronic pain syndrome and somatosensory deficit. Perhaps Charcot was somewhat correct when he believed that hysterical phenomena affecting a hemibody indeed has a dysfunction "in the grey matter of the cerebral hemisphere on the side opposite". ${ }^{3}$

Although organic neurological diagnoses could explain the existence of a hemisensory syndrome, only one patient $(3 \%)$ was diagnosed with a specific neurological diagnosis after substantial diagnostic procedures and follow up periods. Thus, the yield of diagnostic testing in hemisensory syndrome is very low. The explanation for symptoms occurring in remaining patients is unclear. The role of psychological processes such as anxiety, depression, or stress may influence the central nervous system and its perception of sensory stimuli, as has been suggested with increased nociception in CRPS (A Mailis, et al, American Pain Society, 16th annual meeting, New Orleans, 1997). How the role of psychobiological interactions between personality and psychological profiles affects sensory processing functions for one hemiportion of the body only is unclear. Similar clinical presentations have been described in patients with CRPS, ${ }^{8} 15$ chronic pain syndromes (A Mailis, et al, American Pain Society, 16th annual meeting), and fibromyalgia (H H S Kaziyama, et al, IASP 9th world congress in pain, Vienna, Austria, 1999). A high incidence of psychiatric illness was diagnosed in patients with hemisensory impairment and CRPS. ${ }^{15}{ }^{16}$ In one study, $84 \%$ of patients with hemisensory deficit and CRPS had a psychiatric diagnosis including mood disorder in $46 \%$, anxiety disorders in $30 \%$, and substance misuse disorders in $14 \% .{ }^{16}$

In this prospective series, the diagnostic yield for specific aetiology of spontaneous hemisensory syndrome was 3\% and all other remaining patients had a benign prognosis. I advocate strong consideration of background history, neuroimaging, and reinforcement of the nearly uniform benign prognosis for patients with spontaneous hemisensory syndrome. I would not advocate aggressive investigations beyond neuroimaging in this group of patients unless specific historical points indicate further interventions. Psychiatric evaluation may be beneficial in some cases. The cause of 
hemisensory syndrome remains obscure and may relate to a combination of central processing difficulties or psychological factors.

\section{ACKNOWLEDGEMENTS}

The author would like to thank Dr A K Brownell for assistance in editing.

\section{REFERENCES}

1 Charcot J.-M. Lecons sur les maladies du systeme nerveux. Paris: Delahaye, 1872-1873.

2 Ballet G. La domaine de la psychiatrie. Presse Med 1991;10:377-80.

3 Charcot J.-M. Lezioni cliniche dell'anno scolastico 1883-84 sulle malattie del sistema nervoso. [Translated by D Miliotti]. Milan.

4 Weintraub MI. Chronic pain in litigation. What is the relationship? Neurol Clin 1995;13:341-50.

5 Vuileumier P, Chicherio C, Assal F, et al. Functional neuroanatomical correlates of hysterical sensorimotor loss. Brain 2001;124:1077-90.

6 Rolak LA. Psychogenic sensory loss. J Nerv Menal Dis 1988; 176:686-7.
7 Stone J, Zeman A, Sharpe M. Functional weakness and sensory disturbance. J Neurol Neurosurg Psychiatry 2002;73:241-5.

8 Covington EC. Psychological issues in reflex sympathetic dystrophy. In: Janig W, Stanton-Hicks M, eds. Reflex sympathetic dystrophy: a reappraisal. Seattle, WA: IASP Press, 1996:191-216.

9 Kim JS. Pure sensory stroke. Clinical-radiological correlates of 21 cases. Stroke 1992;23:983-7.

$10 \mathrm{Kim}$ JS, Bae YH. Pure or predominant sensory stroke due to brain stem lesion. Stroke 1992;28:1761-4.

11 Bassetti C, Bogousslavsky J, Regli F. Sensory syndromes in parietal stroke. Neurology 1993;43:1942-9.

$12 \mathrm{Kim}$ JS. Lenticulocapsular hemorrhages presenting as pure sensory stroke. Eur Neurol 1999;42:128-31.

13 Bogousslavsky J, Regli F, Uske A. Thalamic infarcts: clinical syndromes, etiology, and prognosis. Neurology 1988;38:837-48.

14 Paciaroni M, Bogousslavsky J. Pure sensory syndromes in thalamic stroke. Eur Neurol 1998;39:211-17.

15 Rommel O, Gehling M, Dertwinkel R, et al. Hemisensory impairment in patients with complex regional pain syndrome. Pain 1999;80:95-101.

16 Rommel O, Malin JP, Zenz M, et al. Quantitative sensory testing, neurophysiological and psychological examination in patients with complex regional pain syndrome and hemisensory deficits. Pain $2001 ; 93: 279-93$. 